\title{
Curzio Malaparte 60 ans après sa mort : états de la question et perspectives
}

\section{Emmanuel Mattiato}

\section{OpenEdition}

\section{Journals}

Édition électronique

URL : http://journals.openedition.org/cei/3274

DOI : $10.4000 /$ cei.3274

ISSN : 2260-779X

Éditeur

UGA Éditions/Université Grenoble Alpes

Édition imprimée

ISBN : 978-2-84310-370-4

ISSN : 1770-9571

Référence électronique

Emmanuel Mattiato, "Curzio Malaparte 60 ans après sa mort : états de la question et perspectives », Cahiers d'études italiennes [En ligne], 24 | 2017, mis en ligne le 28 février 2017, consulté le 27 mars 2021. URL : http://journals.openedition.org/cei/3274 ; DOI : https://doi.org/10.4000/cei.3274

Ce document a été généré automatiquement le 27 mars 2021

(c) ELLUG 


\title{
Curzio Malaparte 60 ans après sa mort : états de la question et perspectives
}

\author{
Emmanuel Mattiato
}

1 Témoin et visionnaire. On peut se réjouir de cet intitulé, choisi par Michèle Coury pour donner son orientation à la journée d'étude qu'elle a organisée à l'Université Grenoble Alpes le 21 mai 2015, en rassemblant des chercheurs de France et d'Italie. Tout Malaparte est contenu dans cette formule concise, comme dans un noyau incandescent : en effet, de son vivant déjà, il fut considéré comme un témoin privilégié des cataclysmes de son époque; quant à son génie visionnaire, ce n'est pas immédiatement ce qu'ont retenu de lui ses contemporains, davantage aveuglés et excédés par ses outrances et sa réputation - tantôt fondée, tantôt erronée, et le plus souvent exagérée - d'impénitent « voltagabbana ».

2 Sans doute fallait-il qu'il mourût et que, après des décennies de célébrité, son nom sombrât pour un temps dans un relatif oubli. Dans le climat bipolaire et manichéen de la guerre froide, on avait en horreur les parcours non linéaires, surtout quand l'intéressé avait osé se compromettre avec le fascisme honni, dont certains croyaient deviner l'épouvantable réincarnation, ou mutation, sous les traits de l'Oncle Sam ${ }^{1}$. À la limite, pouvait-on pardonner ces fautes de jeunesse à Pratolini, Bilenchi et Vittorini, entre autres ; mais cela s'avérait plus difficile concernant Malaparte ${ }^{2}$.

Outre l'hégémonie intellectuelle communiste qui s'instaure après 1945 et qui ne pouvait que nuire à Malaparte en dépit de sa sympathie pour l'Union soviétique ${ }^{3}$, les assimilations instrumentalisées entre fascisme et américanisme qui avaient alors cours allaient forcément aggraver son sort et, après sa mort en 1957, entraver la redécouverte et une sereine étude critique de son œuvre. De plus, cette assimilation durable entre fascisme et américanisme a probablement désamorcé la virulence du discours malapartien à l'encontre d'un certaine Weltanschauung américaine appliquée à l'Europe et au monde, celle-là même qui allait provoquer tant de dégâts géopolitiques au Proche et au Moyen-Orient (surtout après 1990, de la seconde guerre du Golfe aux 
soi-disant printemps arabes) et dont on peine, aujourd'hui encore, à voir l'issue. De fait, la représentation qu'il donne des Américains dans La pelle est des plus corrosives et prolonge, en la désidéologisant, l'américanophobie fasciste des années de guerre ${ }^{4}$; il ne faut pas exclure non plus que l'anti-américanisme qui imprègne ce roman soit une revanche de l'écrivain par rapport à un pays qui l'a en quelque sorte contraint à devenir l'un de ses informateurs secrets au moment de la campagne pour la libération de l'Italie ${ }^{5}$.

Cette hostilité envers la Babylone d'outre-Atlantique n'est pas nouvelle en 1945: hormis qu'elle est une figure topique de la littérature européenne au moins depuis la période décadente et symboliste, que l'on n'oublie pas que le libéralisme apatride anglo-américain avait été vilipendé par la frange la plus antimoderne et barbara du fascisme dès les années $1920^{6}$. Cette composante des squadristi avait pu paraître, quantitativement, minoritaire mais elle avait été suffisamment influente pour irriguer le fascisme espagnol ${ }^{7}$ et avait quelque ressemblance avec certains mouvements de l'aire germanique ou anglo-saxonne préoccupés par l'écologie et technophobes. Malaparte a uni son nom à ce fascisme enraciné et provincial dès la création de la revue Strapaese par Mino Maccari (qu'il ne cesse de protéger tout au long du Ventennio), au point d'en devenir, avec Papini et Soffici, l'un des plus éminents représentants ; et ce, en dépit de sa fascination duelle pour l'autre versant culturel du fascisme, le plus cosmopolite novecentismo ${ }^{8}$.

5 L'antiaméricanisme ne fait que se renforcer dans les milieux intellectuels fascistes dans les années $1930^{\circ}$. Pour d'évidentes raisons d'opportunisme politique, cette critique va croissant après la guerre d'Éthiopie (il s'agit alors de dénigrer l'esprit mercantile anglosaxon en général), surtout parmi les jeunes chemises noires qui n'avaient pas vécu les années « héroïques » de la marche sur Rome et qui, de plus, pouvaient se sentir tentées par l'esprit révolutionnaire et radical du soviétisme ${ }^{10}$; et c'est précisément sur cette «troisième voie » de garage que l'on croise Pratolini, Vittorini et Bilenchi, sans oublier Indro Montanelli, Roberto Pavese, Salvatore Gatto ${ }^{11}$ et, surtout, Berto Ricci, le prolifique directeur de L'Universale ${ }^{12}$ et pilier de la " campagna antiborghese ${ }^{13}$ ", encouragée par le Duce à la veille de la Seconde Guerre mondiale pour ranimer une flamme noire devenue vacillante. L'apogée du consenso et le succès d'image remporté par Mussolini au moment de la proclamation de l'Impero semblent bel et bien révolus. Lorsqu'éclate le nouveau conflit mondial, Malaparte - quoiqu'ayant appuyé, surtout après 1935, la propagande d'État par la $1^{\text {re }}$ série de la revue Prospettive ${ }^{14}$ et par des reportages de circonstance lus par les centaines de milliers de lecteurs du Corriere della Sera - n'a plus qu'à se pencher cruellement au chevet du régime en se faisant, dans Kaputt, le chroniqueur impitoyable de sa déchéance.

Dès mars 1943, au cœur même de la Seconde Guerre mondiale, Malaparte se trouve en Finlande et, dans un article jamais cité et d'une certaine portée prophétique, il a l'intuition que les grands enjeux géopolitiques de l'après-guerre reposeront sur un affrontement titanesque entre États-Unis et URSS ${ }^{15}$. Quelques années plus tard, dans une Italie défigurée par une guerre civile si cruellement «représentée » dans La pelle, force est de reconnaitre que le climat bipolaire de la guerre froide, avant même qu'il ne se répande dans le monde entier, n'est guère propice à la redécouverte de l'œuvre de l'ex-fasciste Malaparte ${ }^{16}$; et ce, même après sa mort ; au mieux, le tolère-t-on comme l'écrivain sulfureux de Kaputt et de La pelle, ou comme un mondain stéréotypé gravitant entre Rome et Capri, conciliant en lui les vices et vertus de la latinité. 
7 Revenir, comme nous venons de le faire, sur ses liens contrastés avec l'Amérique - comprise à la fois comme représentation mytho-politique dysphorique et comme employeur secret d'un intellectuel qui, volens nolens, s'était compromis avec le régime mussolinien - nous permet aussi de rappeler combien tout modèle impérial pouvait, en tant que centre radiant du pouvoir, exercer une fascination sur un auteur n'ayant eu de cesse de fréquenter les hautes sphères dirigeantes et d'explorer les arcanes du politique : dans cette perspective, par exemple, à l'instar du Travailleur d'Ernst Jünger, qui en suit la publication d'un an, le traité Technique du coup d'État (1931) dépasse le simple essai de réflexion historico-politique dans le sillage de Machiavel : c'est aussi, en filigrane, une méditation sur les ressorts les plus secrets de la violence à l'âge des totalitarismes et sur l'essence technique de toute praxis militante ; et, plus globalement, sur la technique comme destin de l'Occident ${ }^{17}$.

8 Le climat libéral et normalisateur de l'Europe d'après-guerre n'est donc pas favorable à l'affirmation de l'œuvre malapartienne, plutôt représentative de cet âge bouillonnant et anticonformiste que fut l'entre-deux-guerres. Même la conversion tardive de l'écrivain au communisme était regardée avec suspicion. On comprend alors que la vingtaine d'années qui s'écoulent après sa mort ne suscitent pas le même élan éditorial et, surtout, critique que celui qui entoure Marinetti à la même époque ${ }^{18}$; sans doute parce que la jeunesse occidentale qu'enthousiasment révolution et utopies était friande de l'expérimentalisme et des provocations du fondateur du futurisme, alpha des avantgardes mondiales.

9 En effet, en dépit de sympathies anciennes pour la Russie rouge et d'une admiration pour le maoïsme, Curzio Malaparte ne parvient pas à séduire les franges antilibérales de gauche (il y eut, certes, la rencontre et la collaboration avec Maria Antonietta Macciocchi, mais elle-même était, en quelque sorte, une hérétique au sein du PCI). Cela s'explique non seulement par la liberté de ton d'un penseur peu en phase avec le «bipolarisme » intellectuel et politique qui précède les années de plomb, mais aussi par le potentiel visionnaire et métaphysique de certaines de ses intuitions : pour ne donner qu'un seul mais fondamental exemple, le topos malapartien du sacrifice du Christpeuple et sa réinterprétation de la tragédie grecque présagent les théories girardiennes du bouc émissaire et du lien primitif entre violence et sacré ; sans compter que sa culture profondément classique, son respect de la tradition et de l'italianité n'avaient rien pour plaire aux jeunes libertaires d'alors.

10 Toutefois, lorsque l'Union soviétique s'effondre au début des années 1990, commencent à se multiplier les études critiques et les rééditions de ses ouvrages, pour ne rien dire des traductions inédites qui fleurissent en France depuis une dizaine d'années ${ }^{19}$. Une brèche s'ouvre dès les années $1980^{20}$, lorsque Giordano Bruno Guerri commet la première biographie approfondie du sulfureux dandy, n'hésitant pas à lui rendre justice sur certains points, tout en noircissant sa légende ou forçant la caricature dans d'autres $\operatorname{cas}^{21}$; cela dit, en dépit de cet intérêt accru pour Malaparte depuis une trentaine d'années, il reste aujourd'hui encore une figure marginale des lettres italiennes. La distance temporelle et idéologique par rapport à l'auteur et à son histoire semble à présent propice non seulement à sa redécouverte et à l'approfondissement de ses écrits dans le strict cadre académique (et loin des banalités d'usage déversées sur son " cas »), mais peut-être aussi à sa canonisation prochaine (comme pourraient le suggérer son inscription aux questions du concours d'agrégation d'italien et le projet 
d'Adelphi et d'autres éditeurs de rééditer ou de traduire son œuvre, voire de proposer des éditions critiques de ses textes emblématiques).

11 Si la question du Malaparte visionnaire est largement ouverte, car elle convoque des catégories d'analyse d'ordre à la fois esthétique et métaphysique, il paraît plus aisé d'aborder le sujet du Malaparte-témoin. À l'instar de Maurice Genevoix, Henri Barbusse, Ernst von Salomon, Erich Maria Remarque, Ernst Jünger, Carlo Emilio Gadda ou Giani Stuparich, sa parabole créatrice et politique débute au lendemain de la Grande Guerre, sur les ruines fumantes d'une Europe dévastée par quatre années de luttes fratricides. Témoin, il le fut jusqu'à l'excès. En 1921, Viva Caporetto!, son premier livre, probablement l'un des plus méconnus consacrés à la Première Guerre mondiale, est immédiatement saisi par les autorités ; et il le sera à chacune de ses rééditions. Souvent malgré lui, quoi qu'on en dise, le scandale accompagne Malaparte à chaque étape de sa vie intellectuelle et sert, en quelque sorte, de caisse de résonance à son témoignage.

Né sous une bonne étoile, ce « témoin vedette de l'histoire ${ }^{22}$ » côtoie très tôt les grandes figures de son temps et, comme Dante avant lui, il utilise la sève de ses exceptionnelles rencontres pour nourrir son écriture. On pressent cela dès le pamphlet Le nozze degli eunuchi, en 1922, et l'art de témoigner s'affirme comme une "marque de fabrique " surtout à partir de Technique du coup d'État, en 1931. Le faux se mêle allègrement au vrai ou, plutôt, au vraisemblable, entendu comme catégorie autorisant toutes les audaces, tous les glissements, toutes les métamorphoses qu'engendre l'illusion poétique et que ses lecteurs superficiels confondaient avec la réalité. Dans ce dernier cas, cette lecture littérale de ses écrits a eu pour principale conséquence de les réduire aux aspects les plus "sensationnels" de Kaputt et de La pelle, dans lesquels on ne voyait qu'une succession de scènes macabres et de chocs esthétiques; ce qui ferait de lui un épigone tardif de Baudelaire et des décadents, alors que son œuvre est autrement plus profonde.

Malaparte était bien conscient de ces glissements entre réalité et fiction, et Michèle Coury, dans ces pages, a raison d'insister sur le fondamental chapitre $\mathrm{x}$ de La pelle ( $\mathrm{La}$ bandiera »), celui qui, mieux que tout autre texte de l'écrivain, donne la clef de sa poétique. Nous sommes à un moment du récit où le narrateur, un Malapartepersonnage semblable au «Dante pellegrino » de la Divina Commedia, est interrogé sur l'idée de vérité dans son roman Kaputt par Pierre Lyautey et le général Guillaume, l'un demandant ironiquement ce qui est vrai dans Kaputt, et l'autre attaquant l'écrivainjournaliste parce qu'il tromperait ses lecteurs. L'ami américain de Malaparte, Jack, répond à sa place, ce qui n'est pas anodin : «Non ha alcuna importanza se quel che Malaparte racconta è vero o falso. La questione da porsi è un'altra; se quel che egli fa, è arte o $\mathrm{no}^{23}$.»

Cela pourrait rappeler a priori le débat rhétorique de l'époque du Tasse sur le vrai et la vraisemblance. On se souvient que le Tasse privilégiait la « verosimiglianza " plutôt que le vrai. Dans le cas de Malaparte qui, lui, s'est bien gardé de codifier sa poétique, on a affaire à une théorie post-baroque où le réel est prétexte à des mirages, à des distorsions, à de fausses perspectives; le récit, toutefois, ne bascule jamais dans l'invraisemblance et l'écrivain sait se tenir agilement sur une ligne de crête. Il est intéressant de remarquer que dans ce débat sur la poétique malapartienne, le narrateur se met en retrait et n'intervient pas, laissant les uns l'accabler pour ses excès, et Jack le défendre en révélant qu'à la base de l'écriture malapartienne, la vérité ou le faux sont 
très secondaires par rapport à l'art (qu'il convient sans doute d'entendre au sens d'art pourl'art).

Cela est bien illustré par la suite du récit. Malaparte-personnage se met alors à raconter le dernier événement sordide dont il aurait été, une fois encore, le témoin : et cela se serait produit quelques minutes plus tôt, au moment même où il prenait son repas avec ses éminents convives. Rapportant les faits comme le ferait un personnage de la « lieta brigata » qui, dans le Decameron de Boccace, se réunit dans la campagne florentine pour fuir la peste, le narrateur déploie sa « novella » sur les hauteurs du champ de bataille et raconte comment il vient de manger une main de goumier tombée dans son couscous. Toute «l'arte » malapartienne tient dans ce récit enchâssé qui s'impose comme une magistrale illustration de cette pure illusion rendue vraisemblable par le génie de l'écrivain démiurge. Les os humains entre les grains de semoule s'avèrent être en fait des os de poulet, et l'illusionniste Malaparte révèle alors à ses convives son «truc » : il a leurré son monde en disposant les os du volatile en leur donnant la forme d'une main.

C'est là un magistral enseignement de poétique romanesque, une leçon sur la capacité qu'a tout écrivain de jouer avec la vérité et sur le pouvoir qu'a l'écriture de métamorphoser le réel. Tout le surréel malapartien découle de cet art particulier consistant précisément à surélever le réel, à le dépasser en le parant de l'illusion du vrai. Andrea Orsucci, dans un stimulant essai sur les liens entre l'écriture malapartienne et la philosophie, a bien remarqué que le « radicale 'sovvertimento' del 'senso' ${ }^{24}$ » auquel recourt l'écrivain a une matrice baroque et se mâtine d'un surréalisme français habilement détourné.

Il faut reconnaître que Malaparte, profitant de son statut de journaliste du Mattino, puis de La Stampa et du Corriere della Sera, n'hésite pas à brouiller les frontières entre réel et surréel, entre actualité et réécriture, réinterprétation. Si tant est que l'on pût décrire objectivement l'actualité, comme le revendiquait une certaine presse traditionnelle anglo-saxonne, la question se complique si l'on prend en examen les vingt premières années de l'activité littéraire malapartienne, coöncidant peu ou prou avec le Ventennio fasciste.

18 Quel que soit le nom qu'on lui prête, la description des "faits", du «vrai », de la « réalité » ou de l'« actualité » sous le fascisme est alors moins régie par une exigence d'objectivité qu'elle n'est une adhésion à une croyance qui s'apparente à une théologie politique; laquelle, au nom de la propagande et des intérêts de la nation, informe le réel et entend conditionner ceux qui prétendent s'en faire les témoins. En ce sens, Malaparte, authentique fasciste hérétique, ne se soumet jamais entièrement à la Weltanschauung mussolinienne, oscillant entre pacifisme impur (Viva Caporetto!, dans ses trois éditions entre 1921 et 1923) et radicalisme syndical-révolutionnaire (la saison militante de La Conquista dello Stato et des textes de théorie corporatiste), louvoyant sans crainte de se contredire entre Strapaese et Stracittà, entre cosmopolitisme et enracinement identitaire.

19 Continuer à envisager les textes malapartiens en leur cherchant une cohérence doctrinale ou, pire, en se chargeant de les évaluer en fonction de notre "moraline " propre (ce que tout chercheur sérieux devrait absolument éviter), ne sera jamais utile à la compréhension de l'écrivain. Aussi, le concernant, se méfiera-t-on de la reductio ad Hitlerum autant que du relativisme idéologique. Il est indéniable que sa condamnation au confino n'est pas simplement due au fait qu'il avait osé diffamer Italo Balbo, quadrumvir de la marche sur Rome : son discours non aligné - et donc, par certains 
égards, critique - a aussi pesé dans la décision qu'a prise Mussolini de le déporter à Lipari ; mais certainement pas au point de faire de lui l'antifasciste qu'il a dépeint dans ses préfaces écrites après la guerre. Les nombreux reportages qu'il effectue pour le Corriere ou le travail de propagande qu'il conduit en dirigeant la $1^{\text {re }}$ série de la revue Prospettive reflètent bien les contradictions du personnage, prompt à se chercher une nouvelle virginité fasciste en appuyant le régime ou en rendant des services à son protecteur, le très puissant Galeazzo Ciano, gendre du Duce et ministre des affaires étrangères.

Toute la production de Malaparte, à la fois littéraire et politique, est pétrie de cette tension entre éléments contraires. Et c'est précisément ce dynamisme alimenté par les opposés qui définit sa stratégie d'écriture. Tout en changeant régulièrement de peau, tel un reptile, il n'en reste pas moins lui-même. Il est loisible à chacun de dépister ces coincidentiae oppositorum, qui sont autant de chausse-trappes pour le chercheur et qui, au premier abord, peuvent décourager toute velléité de parvenir jamais à une critique organique de l'œuvre. Cette dernière est foisonnante, couvrant à la fois la politique, le roman, la nouvelle, la poésie, le cinéma (écriture de scénarios et réalisation), le théâtre, voire l'architecture, la photographie et, dans une infime mesure, la traduction (je pense en particulier à ses traductions pour la $2^{\mathrm{e}}$ série de Prospettive); pour ne rien dire des inédits, des innombrables projets avortés, des écrits inachevées ou autobiographiques.

Cette machine narrative à plusieurs niveaux, qui joue avec les antinomies et se moque des conventions, est opérante tout au long de sa carrière. Pour ne citer qu'un seul exemple, situé au cœur de ce que l'on peut subjectivement considérer comme l'âge d'or de l'écriture malapartienne, cette machine tourne à plein régime dans le cadre de l'incessant échange textuel entre journalisme et roman entre la fin des années 1930 et la fin des années 1940. Aussi, peut-on estimer que les événements fixés dans ses reportages pour le Corriere della Sera durant la Seconde Guerre mondiale sont une version ad usum delphini de son expérience de la guerre que les romans successifs - Il sole è cieco et Kaputt - rendront dans son intégralité et dans sa vérité (nous parlons bien, ici, d'une vérité mouvante, non objective, en constante métamorphose, telle que pouvait la concevoir Malaparte).

En effet, si l'on prend en considération le reportage que Malaparte effectue en Pologne durant l'hiver 1941-1942 (quatre articles publiés entre février et mars 1942), on a en quelque sorte le positif de ces événements qui seront ensuite romancés dans Kaputt et projetés alors en négatif. Nul n'ignore les deux chapitres de Kaputt où Malaparte raconte ses soirées en compagnie du gouverneur général de Pologne Hans Frank, tous se souviennent de ces galeries de portraits psychopathologiques des nazis qui l'entourent ou de la persécution barbare des Juifs du ghetto. Un peu plus de deux ans avant la publication de son roman, dans le dit reportage pour le Corriere, la plupart des personnages mentionnés dans le Kaputt sont déjà présents, à commencer par Hans Frank, le «boucher de Cracovie». Les correspondances, les accords par complémentarité, voire les contre-pieds ou les décalages entre le texte destiné à la presse et celui destiné au roman, sont profondément fascinants et illustrent bien cette manière toute malapartienne d'avancer par contraste, par mues successives, sans vraiment craindre la contradiction. Selon un savant jeu d'imbrications, les contradictions se réalisent diachroniquement, par textes interposés comme nous venons de le rappeler, mais aussi synchroniquement, lorsque, dans le roman, en une même unité narrative, sont décrits des nazis aux goûts raffinés, garants de la culture, 
lesquels cependant se transforment en brutes, la nuit venue, pour aller chasser les " enfants-rats » du ghetto juif ${ }^{25}$.

Les sept contributions de ce numéro des Cahiers d'études italiennes, réparties sur deux sections, explorent les multiples facettes de l'écrivain caméléon. La première section revient aux origines du mythe malapartien, à ses premières années d'activité littéraire dont le pivot est Viva Caporetto!, l'œuvre à laquelle il était le plus attaché, récit inaugural contenant tous les thèmes saillants de sa poétique personnelle. Stéphanie Laporte apporte toutes les précisions nécessaires à la compréhension et à l'interprétation de la genèse de Viva Caporetto!, en revenant sur le contexte à chaque fois différent de ses trois éditions, sur ses oscillations entre pacifisme, marxisme et fascisme, et en montrant ce que ce texte, par son genre hybride, doit au frammentismo littéraire. Emmanuel Mattiato sonde le riche intertexte de ce même ouvrage, en y décelant l'ombre de Marinetti, de Gobetti et, plus surprenant, d'Emerson, le fondateur du transcendantalisme américain, auquel le spiritualisme oceanico doit tant. Il tente simultanément de mettre en lumière certains passages problématiques de l'édition originale de 1921 qui contredisent son orientation générale "pacifiste» et semblent prédisposer son auteur au fascisme. Arianna Camerano revient pour sa part sur l'amitié intellectuelle de Malaparte avec Piero Gobetti, avec lequel il partageait une même vénération pour Georges Sorel, le théoricien du syndicalisme révolutionnaire dont Malaparte développera les thèses dans le contexte du corporatisme fasciste. Le dialogue entre les deux hommes est surtout fertile dans le domaine de l'analyse historique, où, tout en partageant une commune admiration pour certaines figures du Risorgimento, ils diffèrent quant à l'interprétation de la modernité, de la Réforme et de la ContreRéforme. Leur dialogue contrastif culmine dans leur interprétation divergente du phénomène fasciste.

Les contributions de la seconde section sont en revanche consacrées aux écrits de Malaparte à partir de la fin de la Seconde Guerre mondiale. Michèle Coury dévoile un pan de la structure cryptique des grands récits malapartien en partant d'un stylème en apparence anodin disséminé dans les pages de ses deux romans les plus célèbres: en effet, " a voce bassa » inaugure Kaputt et conclut La pelle, et sa récurrence, jamais casuelle, sert le témoignage souvent cruel du narrateur ou de ses interlocuteurs; et, bien plus, cet indice textuel conduit le lecteur à s'interroger sur la dimension sacrificielle, qui est au cœur même des textes et qui est perceptible aussi dans les relations qui se tissent entre humains et animaux symboliques. Laurent Scotto d'Ardino, à travers une analyse de $\mathrm{Il}$ Cristo proibito, le seul film réalisé par Malaparte, s'interroge également sur la symbolique du sacrifice qui imprègne cette œuvre empreinte de néoréalisme ; un néoréalisme très particulier, puisque le réalisateur y insuffle son inspiration personnelle, à la fois théâtrale, religieuse et picturale, condensant dans son long métrage tous ses thèmes les plus chers, à commencer par la puissance révélatrice de la guerre. Lorella Libeccio s'attache quant à elle à étudier comparativement deux anticonformistes majeurs du $\mathrm{xx}^{\mathrm{e}}$ siècle, Malaparte et LouisFerdinand Céline. Abstraction faite d'une lettre du second au premier, il n'existe aucun contact direct entre ces deux monstra de la littérature européenne; toutefois, sur le plan thématique, l'un et l'autre sont unis par une même obsession de la décadence, qui prend chez Céline un pli particulièrement misanthrope et violent, absent des descriptions que donne du peuple l'écrivain toscan. Enfin, Filippo Fonio présente une analyse serrée du théâtre français de Malaparte (à la fois de ses projets achevés et inachevés), en le situant dans les débats idéologiques de son temps - le marxisme 
notamment, par sa critique de la société bourgeoise et décadente - et dans son contexte parisien propre. Comme D'Annunzio, avec lequel les affinités sont nombreuses, il a le souci de ne pas détacher son théâtre de son œuvre romanesque.

En conclusion de cette introduction, nous souhaiterions faire un point non exhaustif sur l'état des recherches et proposer quelques pistes. Si l'on dispose d'une bibliographie assez complète à la fois des textes de Malaparte (y compris de ses articles) et de la critique jusqu'en 1991, placée en appendice du recueil d'actes Malaparte scrittore $d^{\prime} E u r o p a^{26}$, on peut déplorer un certain vide bibliographique concernant les articles de critique universitaire, éparpillés depuis un quart de siècle dans des revues spécialisées dont il n'est pas toujours aisé de trouver la trace. Nous avons dit ce que la redécouverte de Malaparte devait à la biographie de Guerri. Sa traduction en français, associée à la recherche (à l'époque, pionnière) de Michel Ostenc sur la culture fasciste ${ }^{27}$ (lequel consacre de nombreuses pages à l'écrivain toscan), a participé à restaurer le lien ancien entre Malaparte et la France; c'est aussi dans cette lignée que se sont inscrits les travaux de François Livi, Stéphanie Laporte ou Jean-Claude Thiriet.

La biographie de Malaparte que Maurizio Serra publie en 2011 non seulement met à jour celle de Guerri, en y apportant de nombreux éléments inédits mais, écrite en français, elle vaut à son auteur le Goncourt de la meilleure biographie et devient un véritable succès de librairie, suivi d'une édition en italien et en poche. L'ancien représentant de l'Italie à l'Unesco, excellent connaisseur de l'entre-deux-guerres, a eu le mérite de replacer Malaparte dans son contexte culturel européen et mondial et de mettre en lumière le vaste réseau qui composait sa galaxie intellectuelle, tout en fixant, sans doute définitivement, le cadre de ses rapports avec le fascisme. Le Malaparte. Vies et légendes de Serra se situe dans un climat français de rééditions et de nouvelles traductions qui, comme nous l'avions souligné, confortent l'intérêt renouvelé pour l'écrivain italien, dont certains, en 2009, s'étaient étonnés qu'il ait pu autant enthousiasmer Milan Kundera, qui lui réserve de nombreuses pages dans Une rencontre $^{28}$.

Entre Guerri et Serra, une étape fondamentale, qui a permis d'ouvrir le champ de la recherche malapartienne, est marquée par la publication des archives Malaparte en douze épais volumes entre 1991 et 1996 par Edda Ronchi Suckert, sœur de l'écrivain. La plupart des études des vingt dernières années dans ce domaine ${ }^{29}$ s'appuient sur ce matériel qui, en dépit des innombrables coquilles et de l'appareil critique quasi inexistant, pour ne rien dire des reconstitutions à tendance hagiographique qui en faussent la fiabilité, reste une mine d'or pour tout chercheur. Il restera à accomplir un travail philologique titanesque, consistant à confronter cette publication avec les documents d'archives regroupés à la Fondazione Biblioteca di via Senato, présidée par Marcello Dell'Utri, et acquis en 2009, sur fond de scandale, pour la coquette somme de 700000 euros. Outre ce socle, il faudra explorer les autres fonds d'archives disséminés entre France et Italie, à commencer par ceux du Corriere della Sera, essentiels ${ }^{30} \mathrm{car}$, au début des années 1930, ils entérinent le passage de Malaparte à une saison nouvelle de fictions littéraires après une décennie plus fortement ancrée sur les questions plus politiques (cela dit, la politique ne disparaît pas complètement et se mêle aux elzeviri et romans successifs, crescendo après le temps du confino).

Il conviendrait de conclure en disant deux mots des rares, trop rares recueils critiques consacrés à Malaparte. Parmi les spécialistes, un nom émerge parmi d'autres : celui de Luigi Martellini, professeur à l'Università della Tuscia. Il est bien connu pour avoir 
dirigé et annoté les Opere scelte de Malaparte dans la collection «I Meridiani », et ses publications, par leur rigueur, font partie des textes critiques de base. Sans doute pour la première fois, si l'on fait abstraction de quelques articles spécialisés, il explore l'œuvre dans sa totalité et, surtout, d'un strict point de vue littéraire à la fois rhétorique et philologique, là où pendant longtemps les universitaires se contentaient de gloser et de divaguer sur les contradictions du «maledetto toscano » en recyclant tous les lieux communs sur sa vie; et en particulier, évidemment, les plus scandaleux. Les ouvrages scientifiques collectifs restent fort rares dans les années 1980 et $1990^{31}$, et les choses semblent changer durant la décennie 1999-2009 ${ }^{32}$; cependant, ces dernières années, le calme paraît malheureusement être revenu dans les milieux académiques. Il reste donc à espérer que la question «Malaparte " de l'agrégation externe d'italien invite, dans les prochaines années, à la tenue de nouveaux colloques universitaires et suscite de nouvelles vocations. Nous sommes convaincus que le présent numéro des Cahiers d'études italiennes n'est que le prélude à une nouvelle saison faste de recherches malapartiennes.

\section{NOTES}

1. L'époque de la Reconstruction et, surtout, celle qui suit la mort de Malaparte en 1957 n'étaient pas à la nuance, d'autant qu'indéniablement, des ponts avaient été jetés à la fois entre une Italie libérée sous « observation » américaine et la classe dirigeante démocrate-chrétienne, et, de façon plus secrète et sournoise, entre ex-fascistes et services secrets états-uniens. Sans vouloir compromettre outre mesure Malaparte avec les services secrets italiens puis américains, il est indéniable que son nom fut cité dans la sinistre affaire de l'assassinat de Giacomo Matteotti (sur laquelle planent de sombres soupçons de corruption sur des titres pétroliers américains impliquant Mussolini), puis qu'il fut impliqué dans le renseignement avec l'armée de libération américaine au lendemain du débarquement en Sicile: M. Canali, Il delitto Matteotti. Affarismo e politica nel primo governo Mussolini, Bologne, Il Mulino, 1997; Id., "Curzio Malaparte e i servizi segreti americani ", Nuova Storia Contemporeanea, Le Lettere, $\mathrm{n}^{\circ}$ 4, 2009, p. 13-22.

2. Sans doute parce qu'il était de la génération qui, s'étant activement engagée dans la Grande Guerre, avait aussi participé à l'essor du fascisme; du moins, était-ce l'impression de ses contemporains, plus prompts à pardonner aux écrivains de la génération suivante, surtout s'ils étaient des compagnons de route « orthodoxes » du PCI.

3. F. Perfetti, «Curzio Malaparte 1947-1949: contro il fascismo dell'antifascismo», Nuova Storia Contemporanea, Le Lettere, $\mathrm{n}^{\circ}$ 3, 2002, p. 73-92.

4. Avant la guerre, les rapports du fascisme avec les États-Unis étaient plutôt cordiaux, voire chaleureux en dépit de tensions qui vont en s'accentuant après la proclamation de l'Impero, même si celles-ci ne remettront jamais en question la neutralité et une certaine bienveillance américaine dans le jugement des interventions militaires italiennes à partir de 1935. Cf. le classique J.P. Diggins, Mussolini and Fascism. The View from America, Princeton, Princeton University Press, 1972. L'idée générale du livre, qui put faire scandale en son temps, est corroborée par les recherches récentes: S. Luconi, $L a$ «diplomazia parallela». Il regime fascista e la mobilitazione politica degli italo-americani, Milan, Franco Angeli, 2000; S. Luconi et G. Tintori, L'ombra lunga del fascio. Canali di propaganda fascista per gli Italiani d'America, Milan, M \& B 
Publishing, 2004. Voir aussi D. F. Schmitz, The United States and Fascist Italy. 1922-1940, Chapel Hill, University of North Carolina Press, 1988.

5. Sur cette épineuse question, je renvoie à la note 1 de cette introduction.

6. C. Malaparte, Italia barbara, Turin, Gobetti, 1925. C'est sans doute l'œuvre la plus antimoderne de Malaparte, à une époque où l'auteur aurait été attiré par les ors de la Contre-Réforme suivant une approche qui évoque, en l'esthétisant, le maurrassisme (le système politique et la logique intégraliste en moins). En ce sens, le Malaparte strapaesano et barbaro ne rentre pas dans la catégorie de ces intellectuels fascistes obnubilés par l'élan futuriste ou par l'actualisme gentilien, apôtres de ce que Fabio Vander a désigné comme l'« Anti-Italia » (dans son essai très stimulant consacré à la maladive tension révolutionnaire vers le futur et à l'absolutisation de la «nouveauté » qui caractériseraient le mouvement fasciste): F. Vander, L'estetizzazione della politica. Il fascismo come anti-Italia, Bari, Dedalo, 2001.

7. Victoriano Peña a raison de rapprocher le Malaparte d'Italia barbara de l'esprit culturel espagnol de 1898, celui de la "régénération ", qui sera interprété dans un sens antilibéral et antimoderne par Ernest Giménez Caballero, l'un des principaux tenants d'un fascisme espagnol et traducteur de la première anthologie de Malaparte publiée dès 1929 en Espagne. Le catholicisme implicite d'Italia barbara, découlant de l'idée centrale de Controriforma, et les exaltations de la latinité pouvaient bien apparaître en Espagne, à la fin des années 1920, comme un mythe politique fort profitable au jeune fascisme local qui alimentera la Weltanschauung franquiste une dizaine d'années plus tard. V. Peña, Malaparte e la cultura spagnola: dalla scrittura 'barbara' di impegno strapaesano al disincanto antifascista di Kaputt, dans V. Peña (éd.), Scrittura e potere. Intorno all'impegno politico nella letteratura italiana, Rome, Bulzoni, 2010, p.149-189. Voir aussi, plus récemment, l'excellent M. Peloille, Positionnement politique en temps de crise. Sur la réception du fascisme italien en Espagne, 1922-1929, Uzès, Inclinaison, 2015.

8. Sur le complexe de Janus qui tourmente Malaparte, qu'il me soit permis de renvoyer à mon article : Paul Morand e Malaparte. Le tentazioni del cosmopolitismo, dans M. Grassi (éd.), La «Bourse des idées du monde». Malaparte e la Francia, Florence, Olschki, 2008, p. 125-150. Stéphanie Laporte se réfère également au Malaparte bifrons dans son article publié infra.

9. M. Nacci, Le demos et l'or. L'antiaméricanisme en Italie dans les années trente, dans O. Dard et H.-J. Lüsebrink (éd.), Américanisations et antiaméricanismes comparés, Villeneuve-d'Ascq, Presses universitaires du Septentrion, 2008.

10. Sur ce sujet, l'excellente recherche de Parlato reste d'actualité : G. Parlato, La sinistra fascista. Storia di un progetto mancato, Bologne, Il Mulino, 2000.

11. Par exemple S. Gatto, Il borghese, numéro monographique des Quaderni della Scuola di Mistica Fascista, $\mathrm{n}^{\circ}$ 4, 1941. Cf. A. Grandi, Gli eroi di Mussolini. Niccolò Giani e la Scuola di Mistica fascista, Milan, Rizzoli, 2004.

12. P. Buchignani, Un fascismo impossibile. L'eresia di Berto Ricci nella cultura del Ventennio, Bologne, Il Mulino, 1993. À cette liste, il faudrait ajouter le nom du philosophe Ugo Spirito, de la même génération que Malaparte et sans doute l'un des intellectuels les plus proches de lui politiquement. Cf. D. Breschi, Spirito del Novecento. Il secolo di Ugo Spirito dal fascismo alla contestazione, Soveria Mannelli, Rubbettino, 2010.

13. On remarquera que Malaparte avait bien saisi l'esprit séditieux de la jeunesse italienne à la veille de l'entrée en guerre de l'Italie. Le roman inachevé Una tragedia italiana métaphorise les tensions entre la jeunesse en rébellion et le fascisme, devenu système, travers l'opposition (physique et freudienne) entre Paolo et sa mère, la comtesse Tolomei, propriétaire d'un domaine agricole géré de façon exemplaire suivant les orientations du corporatisme fasciste. L'échappée des jeunes dans les profondeurs de la forêt symbolise bien cette révolte sauvage et régressive. C. Malaparte, Una tragedia italiana, in Id., Il ballo al Cremlino e altri inediti di romanzi, Florence, Vallecchi, 1971. Ce roman inachevé fut publié en feuilletons dans les revues Circoli (de juin à décembre 1939) et Raccolta (de janvier à février 1940). 
14. Pour une approche complète de la $1^{\text {re }}$ et de la $2^{\mathrm{e}}$ série de Prospettive: L. Martellini, Le «Prospettive» di Malaparte. Una rivista tra cultura fascista, europeismo e letteratura, Naples, Edizioni Scientifiche Italiane, 2014.

15. C. Malaparte, «La "Porta del Nord" ", Il Corriere della Sera, 23 mars 1943. Indirectement, en ne mentionnant ni l'Allemagne, ni l'Italie parmi les adversaires de demain, il suggère une défaite prochaine de l'Axe.

16. Après la Libération, pour ses contemporains et pour la postérité Malaparte semble condamné à rester l'auteur de Kaputt et La pelle - qui sont, de fait, ses chefs-d'œuvre - alors que sa production des années 1920 et 1930, pourtant fondamentale à tout point de vue, reste dans l'ombre ; et quand elle est rééditée, Malaparte lui adjoint une de ces préfaces qui en dénaturent le sens car elles tendent à le faire passer pour un antifasciste alors qu'il fut surtout un fasciste anticonformiste: c'est le cas, entre autres, de Tecnica del colpo di stato (1931, trad. italienne de 1948), du Sole è cieco (1941, réédité en volume en 1947).

17. La prudence, l'admiration ou la franche critique à l'égard de la technique apparaît comme l'un des grands marqueurs philosophiques des années 1930 et, à ce titre, Technique du coup d'État doit être lu en relation aux questionnements d'intellectuels aussi différents, voire antagonistes, que Gramsci, Costamagna, Spirito, Ortega y Gasset, Valéry, Duhamel, Siegfried, Drieu La Rochelle, Benjamin, Spengler, Jünger, Heidegger, A. Huxley, etc.

18. Les années 1960 furent certes riches en rééditions et textes posthumes, mais la décennie suivante marque un véritable désert éditorial et surtout critique. De fait, dans ce dernier domaine, il n'y a guère que les contributions de Gianni Grana et de Luigi Martellini qui aient gardé quelque intérêt, pour ne pas citer celles, d'ordre plus biographique, de Giampaolo Martelli ou Lorenzo Righi : G. Grana, Malaparte, Florence, La Nuova Italia, 1968 ; L. Martellini, Invito alla lettura di Curzio Malaparte, Milan, Mursia, 1977.

19. C. Malaparte, Viva Caporetto!, trad., préface et notes de Stéphanie Laporte, Paris, Les Belles Lettres, 2012 ; Muss suivi de Le grand imbécile, trad. de C. Cavallera, Paris, Quai Voltaire, 2012 ; C. Malaparte, Voyage en Éthiopie et autres écrits africains, trad. de L. Brignon, Paris, Arléa, 2011 ; C. Malaparte, Le compagnon de voyage, trad. de C. Cavallera, Paris, Quai Voltaire, 2009. Notons que Stéphanie Laporte prépare actuellement une traduction inédite des Battibecchi malapartiens. Dans le même laps de temps, notons aussi les innombrables rééditions de traductions, dont principalement: C. Malaparte, Ces sacrés Toscans, suivi de Deux chapeaux de paille d'Italie, trad. de G. Piroué, Paris, Les Belles lettres, 2014 ; C. Malaparte, Sodome et Gomorrhe suivi de La tête en fuite, trad. de R. Novella et G. Piroué, Paris, Les Belles lettres, 2014 ; C. Malaparte, Journal d'un étranger à Paris, trad. de G. Cabrini, Paris, La Table ronde, 2014 ; C. Malaparte, Ces chers Italiens, trad. de M. Pomès, Paris, Les Belles Lettres, 2013 ; C. Malaparte, Le bal au Kremlin, trad. de N. Frank, Paris, Gallimard, 2013 ; C. Malaparte, La Volga naît en Europe, trad. de J. Bertrand, Paris, Les Belles Lettres, 2012 ; C. Malaparte, Monsieur Caméléon, trad. de L. Allary, Paris, La Table Ronde, 2011.

20. La réévaluation de Malaparte commence en effet au début des années 1980, au même moment où, pour des raisons assez similaires en fait, les sciences politiques reconsidèrent la géopolitique, reléguée depuis 1945 dans le purgatoire académique en tant que discipline universitaire issue du pangermanisme et du fascisme et, comme telle, impropre à expliquer le choc entre communisme et libéralisme. Dans le domaine des lettres, qui nous intéresse, on peut estimer que les réévaluations scientifiques de la question d'une prétendue «culture fasciste » commencent dans les années 1960-1970, grâce aux travaux précurseurs de Luciano De Maria puis de Giovanni Lista sur le futurisme, pour ne rien dire du révisionnisme historique (si âprement contesté) de Renzo De Felice.

21. G. B. Guerri, L'arcitaliano. Vita di Curzio Malaparte, Milan, Bompiani, 1980. Ce succès éditorial est traduit en français un an plus tard, chez Denoël.

22. F. Charbonneau (éd.), Figures de pensée. Vingt-cinq portraits de lucidité et de courage, Montréal, Liber, 2014, p. 182. 
23. C. Malaparte, La pelle, Milan, Mondadori, 1993, p. 269.

24. A. Orsucci, Il «giocoliere d'idee». Malaparte e la filosofia, Pise, Edizioni della Normale, 2015, p. 165. 25. Voir les jeux de miroir décrits dans G. Bàrberi Squarotti, L'allegoria degli orrori della guerra, dans R. Barilli et V. Baroncelli, Curzio Malaparte. Il narratore, il politologo, il cittadino di Prato e dell'Europa, Naples, CUEN, 2000, p. 285-306.

26. V. Baroncelli et C. Santi, Bibliografia dell'opera e della critica, dans G. Grana et V. Baroncelli (éd.), Malaparte scrittore d'Europa, s. 1. [Milan], Marzorati-Comune di Prato, 1991, p. 267-373.

27. M. Ostenc, Intellectuels italiens et fascisme (1915-1929), Paris, Payot, 1983.

28. Dans le recueil Une rencontre, on s'est étonné de l'importance que Kundera attribue à Malaparte à travers un dernier chapitre entièrement consacré à l'analyse de La pelle, en relative opposition (ce qui peut sembler inhabituel) avec Kaputt. D'après nous, le plus fascinant, dans cette étude, c'est que Kundera parle de lui-même à travers Malaparte ; il se reconnaît aisément dans l'écrivain italien, notamment dans cette distinction nette établie entre écrivain engagé et désengagé : non sans simplifier, Kundera écrit que Malaparte est « au courant de tout dans la vie politique, il est mondain, sait briller et séduire, mais il est surtout amoureux de l'art et de la poésie. Il préfère toujours Pétrarque à Garibaldi, et les gens qu'il chérit par-dessus tout sont les artistes et les écrivains. Et parce que Pétrarque représente pour lui plus que Garibaldi, son engagement politique est personnel, extravagant, indépendant, indiscipliné [...] ». M. Kundera, Une rencontre, Paris, Gallimard, 2009, p. 183-184: passage à mettre en perspective avec les souvenirs autobiographiques de Kundera rapportés dans ibid., p. 130-134.

29. C'est le cas, par exemple, de l'excellent G. Pardini, Curzio Malaparte. Biografia politca, TrenteMilan, Luni Editrice, 1998.

30. C'est ce que j'avais commencé à faire en 2003, à travers ma thèse de doctorat consacrée aux écrivains-journalistes du Corriere durant la Seconde Guerre mondiale. Voir aussi E. R. Laforgia, Malaparte scrittore di guerra, Florence, Vallecchi, 2011.

31. Il faut signaler le numéro monographique de Chroniques italiennes, Fosca Mariani Zini (éd.), Université de la Sorbonne Nouvelle-Paris 3, n 44, 1995, et G. Grana et V. Baroncelli (éd.), Malaparte scrittore d'Europa, ouvr. cité.

32. M. Grassi et F. Goti (éd.), Viaggio fra i terremoti. Malaparte e il giornalismo. Atti del convegno, Prato, 12 dicembre 2008, Prato, Biblioteca comunale Alessandro Lazzerini, 2009. M. Grassi (éd.), La «Bourse des idées du monde». Malaparte e la Francia. Atti del convegno internazionale di studi su Curzio Malaparte, Prato-Firenze, 8-9 novembre 2007, Florence, Leo Olschki, 2008. R. Barilli et V. Baroncelli, Curzio Malaparte. Il narratore, il politologo, il cittadino di Prato e dell'Europa, Naples, Cuen, 2000. C. Di Biase (éd.), Curzio Malaparte. La rivolta del santo maledetto, Naples, Cuen, 1999.

\section{AUTEUR}

\section{EMMANUEL MATTIATO}

Université Savoie Mont Blanc 